\title{
MLPM: Machine Learning Package Manager
}

\author{
Xiaozhe Yao \\ xiaozhe.yao@uzh.ch
}

\begin{abstract}
In recent years, the machine learning community has witnessed a rapid growth in the development of deep learning and its application. Unlike other software that can be installed through the package manager, developing machine learning systems usually needs to search for the source code or start from scratch, debug and then deploy to production. It usually costs much for small companies and research institutions to run, test, evaluate, deploy and monitor machine learning system. In this paper, we proposed a machine learning package manager aiming to assist users 1) find potentially useful models, 2) resolve dependencies, 3) deploy as HTTP service. By using the MLPM, users are enabled to easily adopt existing and well-established machine learning algorithms and libraries to their project within few steps. MLPM also allows third-party extensions to be installed, which makes the system customizable according to users' workflow.
\end{abstract}

\section{KEYWORDS}

MLOps, Model Management, Model Sharing, Model Deployment

\section{ACM Reference Format:}

Xiaozhe Yao. 2020. MLPM: Machine Learning Package Manager. In Austin TX '20: Workshop on MLOps Systems, March 4, 2020, Austin, Texas. ACM, New York, NY, USA, 3 pages. https://doi.org/10.1145/nnnnnnn.nnnnnnn

\section{INTRODUCTION}

In the past few years, machine learning has played an important role in artificial intelligence. There appeared a lot of high-level frameworks, such as [1], [4] to reduce the complexity in developing machine learning algorithms. However, the bottleneck still exists in model sharing and deployments. Currently, the main approach to sharing and distributing of machine learning models is redistributing the source code and trained weight file, which causes incompatibilities and brings extra prerequisites knowledge when deploying machine learning services.

To reduce the time and efforts for deploying and sharing models, we proposed and developed MLPM. Currently, it could help users to 1) download packages from git repositories, 2) build installed packages into docker images, 3) start machine learning services as Restful HTTP API inside docker containers or locally, 4) use extensions to deploy to clusters, such as Kubernetes or Swarm. With all these features, users could use a unified interface to call services across many different machine learning services and libraries.

Permission to make digital or hard copies of all or part of this work for personal or classroom use is granted without fee provided that copies are not made or distributed for profit or commercial advantage and that copies bear this notice and the full citation on the first page. Copyrights for components of this work owned by others than ACM must be honored. Abstracting with credit is permitted. To copy otherwise, or republish, to post on servers or to redistribute to lists, requires prior specific permission and/or a fee. Request permissions from permissions@acm.org.

Austin TX '20, March, 4, 2020, Austin, Texas

(c) 2020 Association for Computing Machinery.

ACM ISBN 978-1-4503-9999-9/18/06 .\$15.00

https://doi.org/10.1145/nnnnnnn.nnnnnnn
MLPM plays as the key component in the open source MLOps system named A.I.D and was firstly released on GitHub ${ }^{1}$ in 2018. A video demonstration for the installation, deployment and test for a face detection service is made available on YouTube ${ }^{2}$.

\section{USAGE}

\subsection{Key Concepts}

Solver is the minimal unit of a machine learning service. Each solver is typically a python class that implements the constructor to initialize the model and restore the weight file, and an infer function to receive the input and return predicated output. Solvers can also optionally implement the train method to receive the dataset, train the model on the given dataset and save the weight file.

Package is a set of solvers, combined with pretrained files, configurations and other optional assets. Package is considered to be a set of similar machine learning algorithms. For example, there is a package named "Face Utility", which is a set of algorithms for face-related tasks(face detection, face landmark recognition and face encoding).

Figure 1: Files needed for a package. The dashed boxes are optional files. the dotted boxes are files that will be automatically generated after installation and the rimless boxes indicate files that users need to fill in.

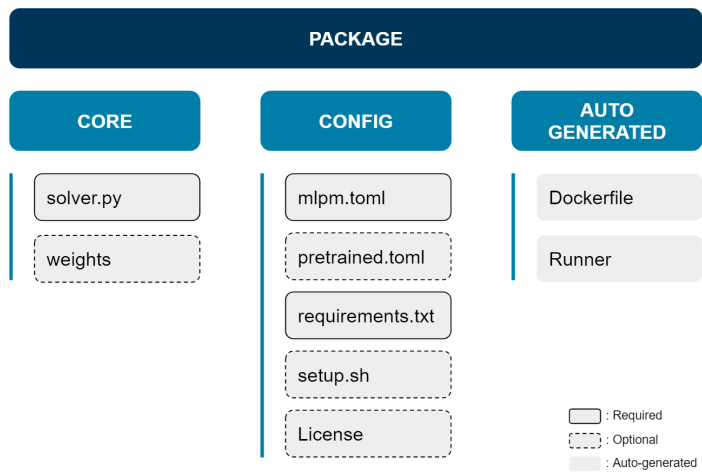

It is straightforward to compose a new package. In Fig.1, we demonstrate what files are needed for the composition, and only two files are mandatory, the solver.py and mlpm.toml. Inside solver, machine learning developers need to define their Solver class by implementing the constructor, infer and optional train methods and indicating where to find them in mlpm.toml. Besides, they can also optionally indicate where to download their trained models in pretrained.toml if the models are not given along with the package. The system will automatically fetch the pretrained weights when installing the algorithms if they are indicated in the pretrained.toml.

\footnotetext{
${ }^{1}$ https://github.com/autoai-org/aid

${ }^{2}$ https://www.youtube.com/watch?v=0TU28hkx7KE
} 
Most dependencies can be installed within the Python Package Manager and requirements.txt but if they need additional installation steps, they need to indicate them inside setup.sh.

Dockerfile and Runner will be automatically rendered for each solver defined in the package after package installation. The Dockerfile indicates what the dependencies are included for the solver, and specifies to run the runner file when starting the container created by the image. The Runner will load the solver and start an HTTP server.

\subsection{Process of Model Deployment}

With MLPM, users can deploy machine learning models in three steps: 1) find an interesting package that is packed as MLPM package on GitHub, 2) install with command line or web-based UI interface, 3) build and deploy to production. The workflow is demonstrated in Fig. 2. MLPM will handle other chores, such as downloading trained weight, generating docker files and serving service.

After the installation, MLPM will save the deployed address where the HTTP server will be listening. Every time MLPM receives users requests to call the inference service, it will redirect the requests to the remote address. By using the redirection, MLPM allows users to monitor the performance, such as running time, QPS, etc.

Figure 2: Process of deploying machine learning services. Users could freely choose to deploy to docker ecosystems or locally and their choice will trigger slightly different steps.

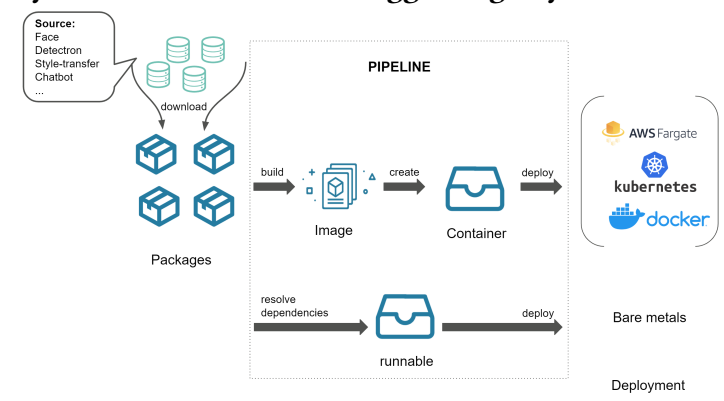

\subsection{Extensions}

There are many advancements in machine learning, such as unified intermediate representation, performance optimizer with the inference engine, robustness certification, etc. We believe it is important for MLOps systems to be compatible with these advancements. Inside MLPM, we allow third-party extensions to interact with packages through hooks which will be triggered when a package is installed, built, deployed, etc. Users could either download third-party scripts or set webhooks to notify the extensions to be performed.

Local Scripts. Third-party scripts can declare their entries in a single script with some placeholders, such as \{\{image_name\}\}, \{\{container_id\}\}. These placeholders will be replaced when the event is triggered. This is designed for actions that require heavy computations, such as model compression, quantization, etc.
Hosted Services. Hosted services will be stored as a webhooks inside MLPM, and will be called through HTTP requests. Thirdparties could receive requests and perform certain actions, such as notification, performance reporting, etc.

\section{CONCLUSION}

Compared with other model markets, we present an open-source package manager for machine learning. It has three main contributions and benefits to the MLOps ecosystem.

- Developers can use GitHub or any other Git-based versioning systems to store, distribute their algorithms, which could turn their researches into products.

- It also enables users to easily download and deploy machine learning services to either bare metals or docker-based ecosystems, such as Kubernetes or Swarm. It could help small companies to reduce their cost in developing machine learning backed systems. Some research institutes may also benefit as they could use it as a quick reproduction of previous research.

- It also allows third-parties to propose extensions for productionlevel use. It could help users adopt recent advancements in deep learning to their production-ready services.

\section{FUTURE WORK}

Currently, MLPM is still at its early stage and being developed heavily. In the near future, we have the following plans:

- Improve the Web User Interface. It is important for users who do not know much about machine learning and docker ecosystem as the web user interface is designed to be simple and easy to use.

- Provide a model hub where users could search and find models more easily. Our package specification could be easily extended to a hub for machine learning models. There are already some model hubs, such as [2], [3], TensorFlow Hub, etc. Compared with them, our planned model hub is frameworkagnostic, extension-enabled and could, therefore, support many more advancements.

- Enrich the resources of models and extensions. Currently, we have implemented several important packages as examples, which can be found at aidmodels ${ }^{3}$. We planned to add more interesting and widely used algorithms as packages, such as AutoML, image caption, etc. Besides of packages, we will also add some model optimization techniques, like quantization as extensions.

\section{REFERENCES}

[1] Martín Abadi, Paul Barham, Jianmin Chen, Zhifeng Chen, Andy Davis, Jeffrey Dean, Matthieu Devin, Sanjay Ghemawat, Geoffrey Irving, Michael Isard, et al. 2016. Tensorflow: A system for large-scale machine learning. In 12th \{USENIX\} Symposium on Operating Systems Design and Implementation (\{OSDI\} 16). 265283.

[2] Ryan Chard, Zhuozhao Li, Kyle Chard, Logan Ward, Yadu Babuji, Anna Woodard, Steven Tuecke, Ben Blaiszik, Michael Franklin, and Ian Foster. 2019. DLHub: Model and data serving for science. In 2019 IEEE International Parallel and Distributed Processing Symposium (IPDPS). IEEE, 283-292.

[3] Ahmed Hosny, Michael Schwier, Christoph Berger, Evin P Örnek, Mehmet Turan, Phi V Tran, Leon Weninger, Fabian Isensee, Klaus H Maier-Hein, Richard McKinley,

\footnotetext{
${ }^{3}$ https://github.com/aidmodels
} 
et al. 2019. ModelHub. AI: Dissemination Platform for Deep Learning Models. arXiv preprint arXiv:1911.13218 (2019).

[4] Adam Paszke, Sam Gross, Francisco Massa, Adam Lerer, James Bradbury, Gregory Chanan, Trevor Killeen, Zeming Lin, Natalia Gimelshein, Luca Antiga, et al. 2019.
PyTorch: An imperative style, high-performance deep learning library. In Advances in Neural Information Processing Systems. 8024-8035. 